\title{
ARMAZENAMENTO DE DOIS TIPOS DE SAPOTI SOB CONDIÇÃO DE AMBIENTE ${ }^{1}$
}

\author{
MARIA RAQUEL ALCÂNTARA DE MIRANDA², FRANCINALMA SOCORRO DA SILVA ${ }^{3}$, RICARDO ELESBÃO \\ ALVES $^{4}$, HELOÍSA ALMEIDA CUNHA FILGUEIRAS ${ }^{4}$ E NÁGELA CRISTINA COSTA ARAÚJO ${ }^{4}$
}

RESUMO - Esse trabalho objetiva avaliar a vida útil de dois tipos de sapoti em temperatura ambiente, com ou sem atmosfera modificada (filme de PVC). O experimento constituiu-se no armazenamento dos frutos, coletados na estação experimental da Embrapa em Paraipaba-CE, em temperatura ambiente $\left(24 \pm 2^{\circ} \mathrm{C}\right.$ e $90 \pm 5 \%$ U.R.) e dispostos em bandejas com ou sem filme de PVC. Dois tipos de sapoti foram avaliados quanto à perda de peso, textura, sólidos solúveis totais, açúcares redutores e totais, acidez total titulável e amido. Os frutos sob atmosfera modificada apresentaram uma menor perda de peso, mas apresentaram uma redução mais rápida na firmeza da polpa. Os dois tipos de sapoti apresentaram um decréscimo no teor de sólidos solúveis, acidez, açúcares solúveis totais e pH e um aumento em açúcares redutores ao longo do armazenamento, independentemente das diferentes atmosferas de conservação. Os resultados indicam que os frutos do tipo II (formato da sapota) possuem uma maior vida útil pós-colheita.

Termos para indexação: Manilkara achras L.; qualidade; firmeza; açúcares solúveis; sólidos solúveis totais, atmosfera modificada.

\section{STORAGE OF TWO TYPES OF SAPODILLA UNDER AMBIENT CONDITION}

\begin{abstract}
This work aims to evaluate the postharvest life of two types of sapodilla at ambient temperature and with or without modified atmosphere (PVC film packing). The experiment consisted in storing fruits, harvested at Embrapa's (Agroindústria Tropical) Experimental Station located in Paraipaba, Ceará, Brazil, under ambient condition $\left(24 \pm 2^{\circ} \mathrm{C}\right.$ and $90 \pm 5 \%$ U.R), on retail trays where half of these trays was wrapped in PVC and the other half, was not wrapped. Fruits of two types were evaluated for weight loss; pulp firmness; total soluble solids; titrable acidity; pH; total soluble sugars, reducing sugars and starch contents. Fruits stored under MA had less weight loss and greater reduction in pulp firmness when compared to those under AA. Decreases in acidity, soluble solids and total sugar contents and $\mathrm{pH}$ were found in both types of fruit during storage under both atmosphere conditions, as well as an increase in reduced sugars content. The type II fruits, had the longest post-harvest shelf-life.
\end{abstract}

Index terms: Manilkara achras L.; quality; firmness; soluble sugars; total soluble solids, modified atmosphere (MA).

\section{INTRODUÇÃO}

O sapoti é originário da América Central, mais provavelmente do Sul do México. Em 1980, Lakshminarayana levantou a existência de nove cultivares de sapoti no Sul da Índia, sete cultivares na India Oriental e doze na Ocidental. O sapotizeiro é uma fruteira muito bem adaptada às condições edafoclimáticas de nosso País, mais especificamente às da região Nordeste. Seu fruto, o sapoti, é reconhecido por seu delicioso sabor adocicado e levemente adstringente, e, na maioria das vezes, é consumido in natura ou na forma de doces, compotas ou geléias.

O sapoti é um fruto muito perecível e, por ser climatérico (Sastry, 1970 e Abdul-Karim et al., 1987), seu amadurecimento sob condições naturais é rápido, o que dificulta sua conservação e comercialização. Como o sapoti é muito apreciado ao natural, há a necessidade de estabelecer-se técnicas de manejo e conservação pós-colheita do fruto para que este possa ser ofertado em mercados mais distantes, com boa qualidade. $\mathrm{O}$ uso de atmosferas modificadas com remoção de etileno e aumento na concentração de $\mathrm{CO}_{2}$, assim como conservação refrigerada com períodos intermitentes de aquecimento mostraram-se úteis para um prolongamento da vida útil pós-colheita do sapoti (Huertas et al., $1999 \mathrm{e}$ Vargas et al., 1999). Pode-se conservar o sapoti por até 2 a 3 semanas, em temperatura entre 12 e $16^{\circ} \mathrm{C}$ (Yahia, 1997).

Um dos problemas na definição de condições de manuseio do sapoti é que existe uma enorme variabilidade dentro da espécie. A heterogeneidade dificulta grandemente um acordo entre os horticulturistas com respeito à classificação do sapoti em variedades. Os fatores de variação da espécie incluem a forma e tamanho da árvore e do fruto, a cor da polpa e o número de sementes (Leon, 1968).

No Brasil, não existem variedades bem definidas de sapoti, sendo os tipos diferenciados entre si pela conformação da copa da planta e dos frutos, em ovalados, arredondados, oblongos, entre outros; mas, de forma geral, todos apresentam o mesmo sabor (Simão, 1971). Em linguagem popular, no Nordeste do Brasil, os frutos de forma oval são chamados de sapoti e os arredondados, de "sapota". Uma pesquisa da Empresa Pernambucana de Pesquisa Agropecuária (IPA) indicou o uso de 10 matrizes para a produção de frutos para consumo ao natural, dentre as quais, a Itapirema-31 mostrou os melhores resultados em produção, peso médio de frutos e época de colheita (Moura \& Bezerra, 1982).

No campo, os dois tipos de sapoti avaliados no presente trabalho foram diferenciados como "sapota" e "sapoti", por causa das semelhanças no formato e tamanho que um desses tipos apresenta com a sapota, fruto arredondado, de outra espécie da mesma família (Sapotacea).

O objetivo deste trabalho foi avaliar a vida útil de dois tipos de sapoti em temperatura ambiente com ou sem atmosfera modificada por filme de PVC.

\section{MATERIAISEMÉTODOS}

Os frutos utilizados são oriundos de um pomar da estação experimental do Vale do Curu, da Embrapa Agroindústria Tropical, no município de Paraipaba, Ceará. Não se sabe ao certo se os frutos utilizados nesse trabalho são realmente variedades diferentes de sapoti, por isso resolveu-se fazer esse estudo comparativo entre ambos. Os frutos de formato oval são chamados de sapoti tipo I e os arredondados, parecidos com a sapota, de sapoti tipo II. Ambos foram obtidos de plantas originadas da matriz Itapirema-31 do IPA. O experimento foi instalado em 20-08-1999, e a colheita deu-se quando os frutos atingiram o estádio de maturação "de vez" e com a casca sem a textura arenosa. Após serem colhidos, os frutos foram levados para o laboratório de Pós-colheita da Embrapa Agroindústria Tropical, em Fortaleza, Ceará, onde se seguiram $\mathrm{o}$ armazenamento e as avaliações.

Os dois tipos de frutos, denominados daqui em diante apenas de sapoti tipo I e II, foram avaliados no dia da colheita e durante o armazenamento sob atmosfera ambiente e modificada. A atmosfera modificada de conservação consistiu no uso de um filme do tipo PVC (cloreto de polivinila com poros de $12 \mu$ ), transparente e auto-aderente, revestindo os frutos colocados em bandejas de isopor. Foram colocados quatro frutos por bandeja, e metade das bandejas foi envolvida com o filme de PVC. O tempo de armazenamento foi de 12 dias, em temperatura de $24 \pm$ $2^{\circ} \mathrm{C}$ e umidade relativa de $90 \pm 5 \%$. As avaliações foram realizadas em seis época: $0 ; 3 ; 6 ; 8 ; 10$ e 12 dias.

Os frutos de cada tratamento (atmosfera de armazenamento e tipo de fruto) foram analisados quanto: a perda de peso, utilizando-se

1 (Trabalho 180/2001). Recebido: 13/11/2001. Aceito para publicação: 21/08/2002 - Apoio: PADFIN/CNPq e CAPES

2 Aluna de Pós-graduação pela Universidade Federal do Ceará. e-mail: raquel_amiranda@yahoo.com.br

3 Aluna de Pós-graduação pela Universidade Federal da Paraíba

4Embrapa Agroindústria Tropical, Caixa Postal 3761, CEP 60511-110, Fortaleza, Ceará. e-mail: heloisa@ cnpat.embrapa.br. Tel: 852991848 
uma balança semi-analítica com $0,1 \mathrm{mg}$ de precisão; a textura, através de um texturômetro eletrônico; o teor de sólidos solúveis totais (SST) após filtração com papel de filtro, utilizou-se um refratômetro digital da marca ATAGO PR-101; o conteúdo de acidez total titulável (ATT) foi obtido por titulação com $\mathrm{NaOH}$ 0,1N (IAL, 1985); a relação SST/ATT foi obtida através do quociente entre as duas análises; o pH, utilizando-se de um potenciômetro com membrana de vidro (AOAC, 1992); a concentração de açúcares solúveis totais, foram medidos pelo método da antrona (Yemn \& Willis, 1954); os açúcares redutores, segundo Miller (1959), e o conteúdo de amido, segundo AOAC (1992). A senescência foi avaliada de acordo com o aparecimento de sinais como incidência de manchas, infecção por fungos e enrugamento. Os frutos foram considerados senescentes quando apresentavam um ou mais desses sintomas. Adotou-se uma escala subjetiva, com notas variando de 0 a 4 , com base na porcentagem dos frutos afetados: $0=$ ausência de sintomas; $1=1$ a 15 $\% ; 2=16$ a $30 \% ; 3=31$ a $45 \%$; e $4=$ mais de $45 \%$ dos frutos afetados.

$\mathrm{O}$ experimento foi realizado segundo um delineamento experimental inteiramente casualizado, em esquema fatorial $2 \times 2 \times 6$ (tipo de sapoti $\mathrm{x}$ atmosfera $\mathrm{x}$ tempo) e 3 repetições sendo submetido à análise de variância. Quando houve interação significativa entre os fatores, fez-se análise de regressão polinomial.

\section{RESULTADOS E DISCUSSÃO}

Os frutos apresentaram uma redução de peso quando em atmosfera ambiente, mas muito pouca diferença foi observada sob atmosfera modificada (Figura 1), durante o armazenamento. Sob atmosfera ambiente, sapoti do tipo I perdeu significativamente mais peso que o do tipo II. A perda de peso dos frutos sob atmosfera modificada foi muito pequena e, por isso, não comprometeria o valor comercial dos frutos. Os frutos do tipo II sob atmosfera ambiente perderam pouco peso e, portanto, sob este aspecto, seriam considerados mais aceitáveis para comercialização. Mohamed et al. (1996) observaram que a perda de peso do sapoti em atmosfera modificada com filme de PVC foi menor em temperaturas de armazenamento mais baixas. Esses autores constataram que os frutos envoltos em filme de PVC, armazenados por 2 semanas sob temperatura de $15^{\circ} \mathrm{C}$, perderam $1,2 \%$ de peso, enquanto os frutos-controle perderam $8,6 \%$.

Em relação ao teor médio de sólidos solúveis (SST), não houve diferenças significativas entre as diferentes atmosferas utilizadas nem entre os tipos de frutos. Observou-se apenas uma redução com o tempo de armazenamento, de 26 para $21^{\circ}$ Brix. Esse comportamento do sapoti em reduzir a quantidade de sólido solúveis totais, durante o armazenamento, já havia sido notado em publicações anteriores (Flores \& Rívas, 1975)

Foram observadas variações na acidez total titulável (ATT) nos dois tipos de frutos, porém essas variações não foram influenciadas pela condição de atmosfera (Figura 2). Observou-se apenas que a ATT foi ligeiramente mais elevada no tipo I que no tipo II. A acidez desses frutos equipara-se à das cultivares mexicanas SCH-02 e SCH-03 (Lakshminarayana, 1980 e Vélez-Colón et al., 1989). Não foram observadas variações importantes com relação à palatabilidade (SST/ATT), razão pela qual o gráfico correspondente não é apresentado. Os índices de 111,17 no tipo I e 133,44 no tipo II sugerem que o sabor doce é fortemente predominante nesses frutos, podendo mesmo impedir a percepção do sabor ácido.

Tanto no sapoti do tipo I quanto no do tipo II, observou-se um pequeno decréscimo no $\mathrm{pH}$, de 5,3 para 4,9, sem diferenças entre os tratamentos. Essa redução no pH concorda com a redução na acidez total titulável, resultante do consumo dos ácidos orgânicos no metabolismo celular, principalmente o respiratório. Os valores de $\mathrm{pH}$ concordam com os valores encontrados por Vélez-Colón et al. (1989), em experimentos com quatorze cultivares plantadas em Porto Rico.

Os dois tipos de sapoti apresentaram uma grande redução na firmeza da polpa ao longo do armazenamento, sendo que essa redução ocorreu mais rapidamente sob atmosfera modificada (Figura 3).
O teor de açúcares solúveis totais reduziu ligeiramente durante o armazenamento, de 17,8 para $16,3 \%$, independentemente do tipo de fruto e da condição de atmosfera. O sapoti maduro armazena seus açúcares solúveis tanto na forma de glicose quanto na de frutose (Lakshminarayana, 1980, e Lakshminarayana \& Subramanyam, 1966). O conteúdo de açúcares redutores aumentou durante os 12 dias de armazenamento (Figura 4), o tipo I apresentou uma concentração final de açúcares redutores de $13,4 \%$ e $12,2 \%$ e o tipo II de $13,6 \%$ e $12,3 \%$ sob atmosfera ambiente e modificada, respectivamente. Nesse experimento, observou-se que a concentração de amido, aproximadamente $2,4 \%$, ficou praticamente inalterada em ambos os frutos sob as duas atmosferas. O sapoti apresentou esse comportamento ímpar, onde a quantidade de amido não diminuía conforme a quantidade de açúcares redutores aumentava. Estudos sobre as alterações bioquímicas e fisiológicas que ocorrem ao longo do desenvolvimento, poderiam explicar esse comportamento da concentração de amido no sapoti.

Pouco tempo após o climatério, o sapoti começa a senescer, e um dos principais sintomas de senescência é um característico aroma alcoólico. Além da senescência em si, um aroma alcoólico poderia sinalizar uma fermentação anaeróbica resultante da embalagem em atmosfera modificada; isso resultou na avaliação de qualquer mudança desagradável no aroma como sinal de senescência. Tanto no tipo I quanto no tipo II, foram observados sinais de senescência durante o armazenamento, independentemente da atmosfera em que se encontravam, embora esses sinais tenham sido mais acentuados no tipo I (Figura 5). Os frutos do tipo II permaneceram em melhores condições até o final das observações, pois não manifestaram sintomas com nota superior a 1 .

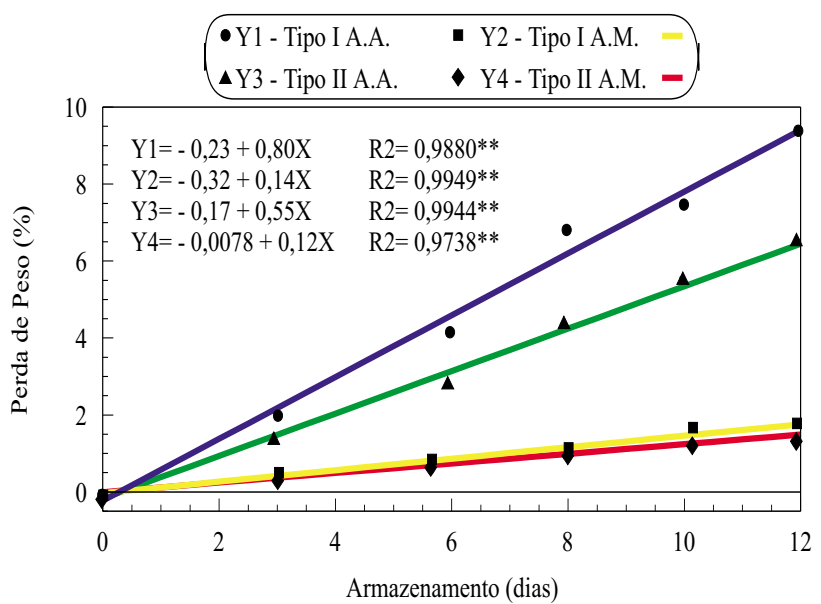

FIGURA 1 - Perda de peso em sapoti dos tipos I ("sapoti") e II ("sapota") associados a atmosfera modificada (A.M) ou não (A.A) e armazenados a $24 \pm 2^{\circ} \mathrm{Ce} 90 \pm 5 \%$ U.R (Fortaleza, 1999).

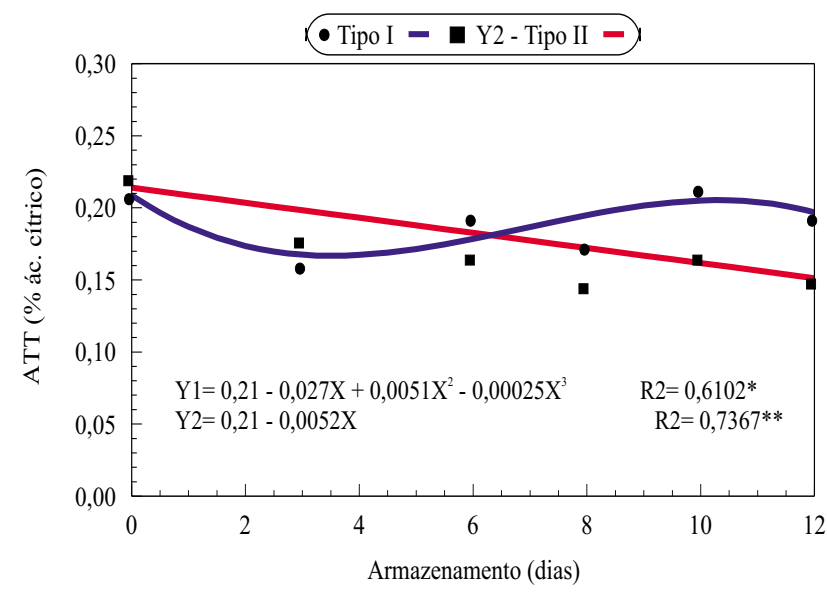

FIGURA 2 - Acidez total em sapoti dos tipos I ("sapoti”) e II ("sapota") associados a atmosfera modificada (A.M) ou não (A.A) e armazenados a $24 \pm 2^{\circ} \mathrm{Ce} 90 \pm 5 \%$ U.R (Fortaleza, 1999). 


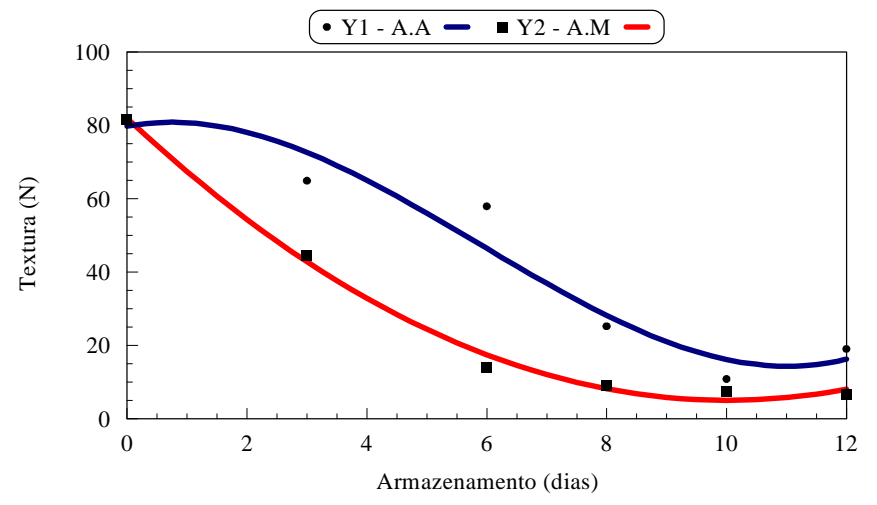

FIGURA 3 - Textura de polpa nos dois tipos de sapoti associados a atmosfera modificada (A.M) ou não (A.A) e armazenados a $24 \pm 2^{\circ} \mathrm{Ce} 90 \pm 5 \%$ U.R (Fortaleza, 1999).

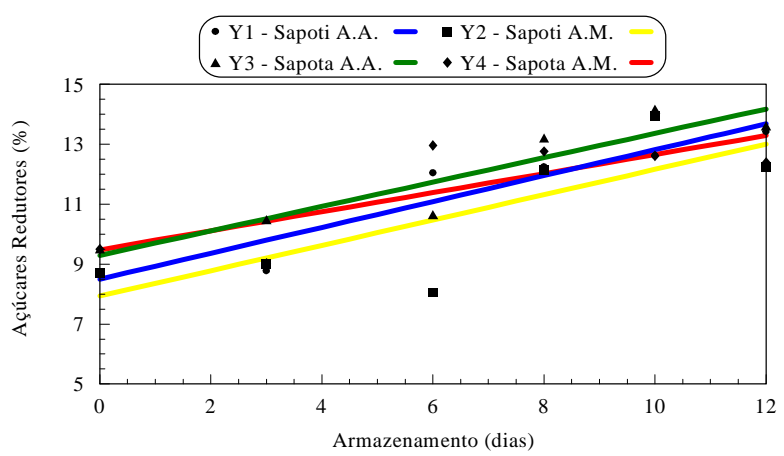

FIGURA 04 - Açúcares Redutores em sapoti dos tipos I (“sapoti”) e II ("sapota") associados a atmosfera modificada (A.M) ou não (A.A) e armazenados a $24 \pm 2^{\circ} \mathrm{C} \mathrm{e} 90 \pm 5 \%$ U.R (Fortaleza, 1999).

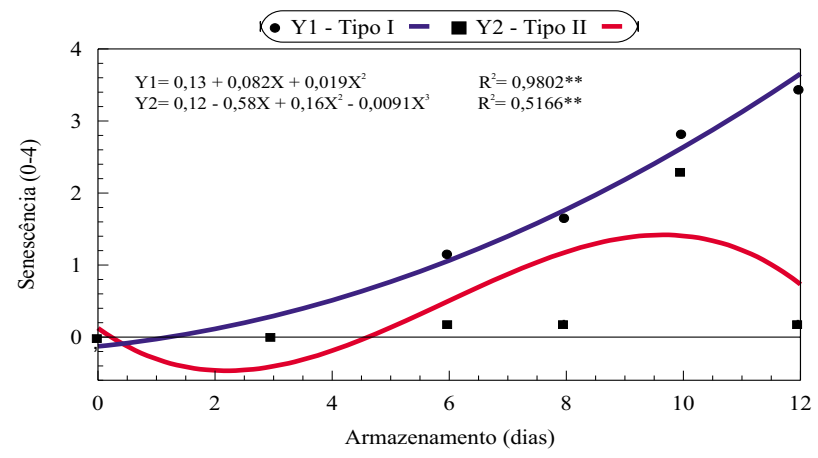

FIGURA 5 - Senescência em sapoti dos tipos I ("sapoti") e II ("sapota") associados a atmosfera modificada (A.M) ou não (A.A) e armazenados a $24 \pm 2^{\circ} \mathrm{C}$ e $90 \pm 5 \%$ U.R (Fortaleza, 1999).

\section{CONCLUSÃO}

Os resultados indicam que os frutos do tipo II, aqueles com o formato da sapota, possuem uma maior vida útil pós-colheita. Levandose em consideração que esses frutos (tipo II) apresentam, também, uma maior uniformidade em tamanho e aspecto, que os do tipo I, essa informação poderia ser útil para os produtores de sapoti. As pequenas diferenças observadas entre os dois tipos de sapoti estudados devem-se, provavelmente, ao fato de esses frutos serem provenientes de plantas que tiveram a mesma origem. A grande variabilidade geralmente obser- vada entre os frutos de sapotizeiro explicaria as diferenças morfológicas e fisiológicas de "vida de prateleira", observadas neste trabalho.

\section{REFERÊNCIASBIBLIOGRÁFICAS}

ABDUL-KARIM,M.N.B.; TARMIZI,A.S.; BAKAR,A.A. The physicochemical cahages in ciku (Achras sapota) of jantung variety. Pertanika, Serdang, v.10, n.3, p.277-282, 1987

ASSOCIATION OF OFFICIAL ANALYTICAL CHEMISTRY (AOAC). Official methods of Analysis of the Association of Official Analytical Chemistry, Washington, 1992. p. 1115.

FLORES G.,A.; RÍVAS,D. Estudios de maduracion y almacenamiento refrigerado de níspero (Achras sapota). Fitotecnia Latinoamericana ,v.11, n.1, 1975, p.43-51.

HUERTAS, G.G.C.; MORENO, N.G.N.; SAURI, D.E. Conservacion refrigerada de chicozapote com calentamiento intermitente. In: CONGRESSO DE HORTICULTURA, 8, 1999, Anais... p.259.

INSTITUTO ADOLFO LUTZ (IAL). Normas analíticas, métodos químicos e físicos para análise de alimentos. 3. ed. São Paulo, 1985 v.1, p.533.

LAKSHMINARAYANA, S., Sapodilla and Prickly Pear. In: NAGY, S.; SHAW, P.E. Tropical and subtropical fruits. Composition, properties and uses. Westport: Avi publishing, 1980. p.415-441.

LAKSHMINARAYANA, S.; SUBRAMANYAM, H. Physical, chemical, physiological changes in sapota fruit (Achras sapota) during development and ripening. Journal of Food Science and Technology, Mysore, v.3, 1966, p.151-154.

LEON, J. Fundamentos botánicos de los cultivos tropicales., San José: IICA, 1969. p. 244-249.

MILLER, G.L.,. Use of dinitrosalicylic acid reagent for determination of reducing sugars. Analytical Chemistry, Washington, v 31, 1959, p.426-428.

MOHAMED, S.; TAUFIK, B. ; KARIM, M.N.A,. Effects of modified atmosphere packaging on the physicochemical characteristics of cyku (Achras sapota) at various storage temperatures. Journal of the Science of Food and Agriculture, Chichester, v.70,1996, p.231-240.

MOURA, R.J.M.; BEZERRA, J.E.F. Instruções técnicas do IPA: Cultivo do sapotizeiro (Achras zapota) em Pernambuco. IPA, 1982. p.3-8.

POPENOE, W. Importantes frutas tropicais. União Panamericana. Departamento de Cooperação Agrícola, União das Repúblicas Americanas. 1989. n.10, p.13-16.

SASTRY, M.V. Biochemical studies on the physiology of sapota. Part III: Minor chemical changes. Indian Food Packer, Calcutta, n.24, 1970, p.20-23.

SIMÃO, S. Manual de fruticultura. São Paulo: CERES, 1971. p.500-508.

VARGAS, L; CENTÚRION, A.; TAMAYO, J. ; SAURI, E. Efecto de las bajas temperaturas sobre las principales características de calidad em frutos de chicozapote (Achras sapota). In: CONGRESSO DE HORTICULTURA, 8., 1999, México. Anais... p.259

VÉLEZ-COLÓN, R.; CALONI, I.B.; MARTÍNEZ GARRASTAZU, S. Sensorial and chemical evaluation of sapodilla (Achras sapota) varieties trials in Puerto Rico. Journal of Agriculture of the University of Puerto Rico, Rio Predras, v.73, n.3, 1989, p. 255-264.

YAHIA, E.M. Modified and controlled atmopheres for tropical fruits. Horticultural Reviews, New York, v.22, 1997, p.123-183.

YEMN, E.W. ; WILLIS, A.J. The stimation of carbohydrate in plant extracts by anthrone. The Biochemical Journal, London, v.57, 1954, p.508-514. 